\title{
Uma aplicação da Extrapolação de Richardson*
}

\author{
$\underline{\text { Gustavo B. Vieira }}^{\dagger} \quad$ Denismar A. Nogueira \\ Instituto de Ciências Exatas, ICEx, UNIFAL-MG, \\ 37130-000, Alfenas, MG \\ E-mail: gborgesvieira@gmail.com, denismar@unifal-mg.com.br
}

\section{RESUMO}

Em muitos processos numéricos fazem-se aproximações da forma $M-N(h)=K_{1} h+K_{2} h^{2}+$ $K_{3} h^{3}+\cdots$, em que $M$ é um valor desconhecido, $K_{1}, K_{2}, K_{3}, \ldots$, são constantes conhecidas e $N(h)$ é uma aproximação de $M$ que depende de $h$. Isso sugere que quanto menor for o valor de $h$, e quanto maior for o índice $n$, para o qual $K_{i}=0$, quando $i \leq n$, melhor será a aproximação de $M$, como afirmam em [1]. O valor de $n$ é conhecido como a ordem da aproximação.

A forma acima de aproximação é conhecida como Extrapolação de Richardson, e é capaz de aumentar a ordem de fórmulas para obter aproximações mais eficientes. Nesse processo, $h$ é denominado passo, e este valor pode ser alterado sem que se altere o valor de $M$, dessa forma, as aproximações são feitas admitindo valores de $h$ cada vez menores, de forma que se combinem as fórmulas de $M$ para gerar aproximações de ordem cada vez maior.

O objetivo deste trabalho é apresentar uma aplicação da Extrapolação de Richardson em um método numérico para auxiliar na aproximação de zeros de funções. Esta aplicação foi desenvolvida em um projeto de Iniciação Científica cujo o título é Integração numérica em várias variáveis e o Método Monte Carlo, no qual são feitos estudos de alguns processos numéricos.

A metodologia consistiu no desenvolvimento de algoritmo computacional em linguagem $\mathrm{R}$ para a obtenção de aproximações numéricas. Nesse trabalho foi aplicado o método para a aproximação de derivadas, a partir da fórmula de diferença centrada na equação apresentada por [1] na aproximação de $M=f^{\prime}\left(x_{0}\right)$ obtém-se:

$$
f^{\prime}\left(x_{0}\right)=\frac{1}{2 h}\left[f\left(x_{0}+h\right)-f\left(x_{0}-h\right)\right]-\frac{h^{2}}{6} f^{\prime \prime \prime}\left(x_{0}\right)-\frac{h^{4}}{120} f^{(5)}\left(x_{0}\right)-\cdots
$$

A fórmula de $N_{i}(h)$ para aproximar a derivada acima é dada por:

$$
N_{1}(h)=\frac{1}{2 h}\left[f\left(x_{0}+h\right)-f\left(x_{0}-h\right)\right]
$$

Substituindo $h$ por $\frac{h}{2}$ em $N_{1}(h)$ o valor de $f^{\prime}\left(x_{0}\right)$ será:

$$
f^{\prime}\left(x_{0}\right)=N_{1}\left(\frac{h}{2}\right)-\frac{h^{2}}{24} f^{\prime \prime \prime}\left(x_{0}\right)-\frac{h^{4}}{1920} f^{(5)}-\cdots
$$

Para prosseguir, é preciso fazer as combinações necessárias para eliminar o termo que contém $h^{2}$. Subtraindo a primeira fórmula de $f^{\prime}\left(x_{0}\right)$ desta segunda fórmula multiplicada por 4 . Assim,

\footnotetext{
${ }^{*}$ O presente trabalho foi realizado com o apoio financeiro da Fundação de Amparo a Pesquisa do Estado de Minas Gerais (FAPEMIG) e do Conselho Nacional de Desenvolvimento Científico e Tecnológico (CNPq).

${ }^{\dagger}$ Bolsista de Iniciação Científica PIBIC/CNPq.
} 


$$
3 f^{\prime}\left(x_{0}\right)=4 N_{1}\left(\frac{h}{2}\right)-N_{1}(h)+\frac{3 h^{4}}{480} f^{(5)}\left(x_{0}\right)+\cdots
$$

Dividindo a fórmula por 3, ficará:

$$
f^{\prime}\left(x_{0}\right)=\frac{1}{3}\left[4 N_{1}\left(\frac{h}{2}\right)-N_{1}(h)\right]+\frac{h^{4}}{480} f^{(5)}\left(x_{0}\right)+\cdots
$$

Desse modo,

$$
N_{2}(h)=N_{1}\left(\frac{h}{2}\right)+\frac{N_{1}\left(\frac{h}{2}\right)-N_{1}(h)}{3} .
$$

Dando continuidade a esse procedimento, será obtido para cada iteração $i=2,3,4, \ldots$ uma aproximação da ordem de $2 i$. Para implementar o algoritmo em linguagem $\mathrm{R}$ foi preciso chegar na fórmula geral destas combinações. Dessa forma, a expressão geral para $N_{i}(h)$ será:

$$
N_{i}(h)=N_{i-1}\left(\frac{h}{2}\right)+\frac{N_{i-1}\left(\frac{h}{2}\right)-N_{j-1}(h)}{4^{i-1}-1}
$$

Uma das aplicações deste método é o cálculo aproximado de derivadas de funções cujas expressões são complicadas e que necessitam de grande precisão. Este resultado pode ser usado no Método de Newton-Raphson, como afirmam em [2], para aproximação de zeros de funções que tem a forma:

$$
x_{n+1}=x_{n}-\frac{f\left(x_{n}\right)}{f^{\prime}\left(x_{n}\right)},
$$

em que $x_{0}$ é uma aproximação da solução da equação $f(x)=0$.

Sendo assim, ao se aproximar os zeros de funções que possuem expressões complicadas através do método de Newtow-Raphson, utilizando a Extrapolação de Richardson, não será necessário o cálculo analítico de sua derivada. O resultado foi o desenvolvimento de um algoritmo em linguagem $\mathrm{R}$ com agilidade e precisão na aproximação de derivadas de funções para utilização neste método que aproximam zeros de funções, uma vez que o desenvolvimento de métodos para o cálculo formal de derivadas é de difícil implementação, mesmo em uma linguagem que permita cálculos algébricos, o que não é possível em linguagem R. Portanto, facilitar a aproximação numérica de zeros de funções com agilidade e precisão é fundamental nesse tipo de cálculo.

Palavras-chave: Extrapolação, Aproximação Numérica, Derivada

\section{Referências}

[1] L.B. Burden, J.D. Faires “Análise Numérica”, Cengage - Learning, São Paulo, 2008.

[2] M.A.G. Ruggiero, V.L.R. Lopes "Cálculo Numérico, aspectos teóricos e computacionais”, Pearson, São Paulo, 1996. 